\title{
Neurofibrosarcoma of the vagus nerve
}

\author{
P. A. CORRIS \\ M.B., M.R.C.P. \\ Regional Cardiothoracic Centre, Freeman Hospital, Newcastle upon Tyne NE7 7DN
}

\section{Summary}

A patient whose symptoms of cough and intermittent hoarseness were due to a neurofibrosarcoma of the vagus nerve is describe 1 . Attention is drawn to the rarity of the tumour and a short review of the pathology and treatment of neurofibrosarcoma is discussed.

KEY WORDS: neurofibrosarcoma, vagus nerve, neurofibromatosis.

\section{Introduction}

Neurofibrosarcomata comprise only $5 \%$ of malignant tumours of connective tissue and may arise either independently or in association with neurofibromatosis (Von Recklinghausen's disease) (Russell et al., 1977).

The patient described here showed interesting clinical features due to a tumour in an unusual site.

\section{Case history}

The patient, a 24-year-old man, presented initially in 1974 with a subcutaneous tumour in the right popliteal fossa; general examination revealed 'café au lait' pigmentation on the back of the trunk and histological examination of the excised tumour confirmed that it was a neurofibroma. In 1978 he developed a spastic tetraparesis and myelography revealed compression of the cervical cord. A cervical laminectomy was performed and a further neurofibroma originating from the second cervical nerve root was excised. Postoperatively the patient recovered completely.

He was readmitted in 1980 for investigation of a dry irritating cough of 2 years' duration and intermittent hoarseness for 2 weeks. He was a non-smoker and had no other respiratory symptoms. A chest Xray showed widening of the superior mediastinum and a lateral view demonstrated an anteriorly placed mass (Fig. 1). A chest X-ray taken in 1978 was reviewed and was normal. On examination he showed widespread 'café au lait' spots and multiple small subcutaneous neurofibromata. There were no abnormal physical signs in the chest or cardiovascular system, abdomen or central nervous system.
Mediastinoscopy was performed and a mass to the right of the trachea was biopsied. Histology suggested neurofibrosarcoma, and thoracotomy was performed.

At operation there was a smooth, hard tumour's $9 \mathrm{~cm} \times 4 \mathrm{~cm} \times 3.5 \mathrm{~cm}$ in the superior mediastinumb behind the superior vena cava extending from the azygos vein into the roof of the neck, arising from theo vagus nerve. The intrathoracic vagus was resecte ${ }^{\circ}$ and the neck then explored via a collar incision. The cervical vagus, which was similarly involved b $\vec{y}$ tumour throughout its length, was also resecteds Histology confirmed neurofibrosarcoma with closely packed pleomorphic cells showing numerous mitoseg with areas of proliferating spindle shaped cells and necrosis.

Postoperatively he made an uneventful recovery and his troublesome cough disappeared.

He presented 12 months later with external tra cheal obstruction secondary to a large local recur rence. However, a remission in tumour size with relief of symptoms has been gained using a combina $\frac{D}{D}$ tion of cyclophosphamide, adriamycin, vincristine and dacarbazine.

\section{Discussion}

Neural tumours in the mediastinum account foㅡㅡㄹ between 19 and $39 \%$ of all intrinsic mediastinab lesions (Strickland and Wolverson, 1974). Typically. they arise in the posterior mediastinum rather tharb anteriorly as occurred in the patient described here They usually originate from either an intercostab nerve root or occasionally from the sympathetic chain, and tumours of the vagus nerve are very rare Strickland and Wolverson (1974) found a total of 21 tumours of the intrathoracic vagus recorded in the literature of which only one was a neurofibrosarN coma. The majority were detected as incidental find స్ట ings on chest $\mathrm{X}$-ray. Patients presenting with symp-o toms all had tumour on the proximal part of the nerve, as in the present case. Hoarseness was a presenting symptom in 3 patients and cough in only one, although 3 patients whose tumour was detected as an incidental finding later admitted to cough. The cougho may have occurred because of direct stimulation 

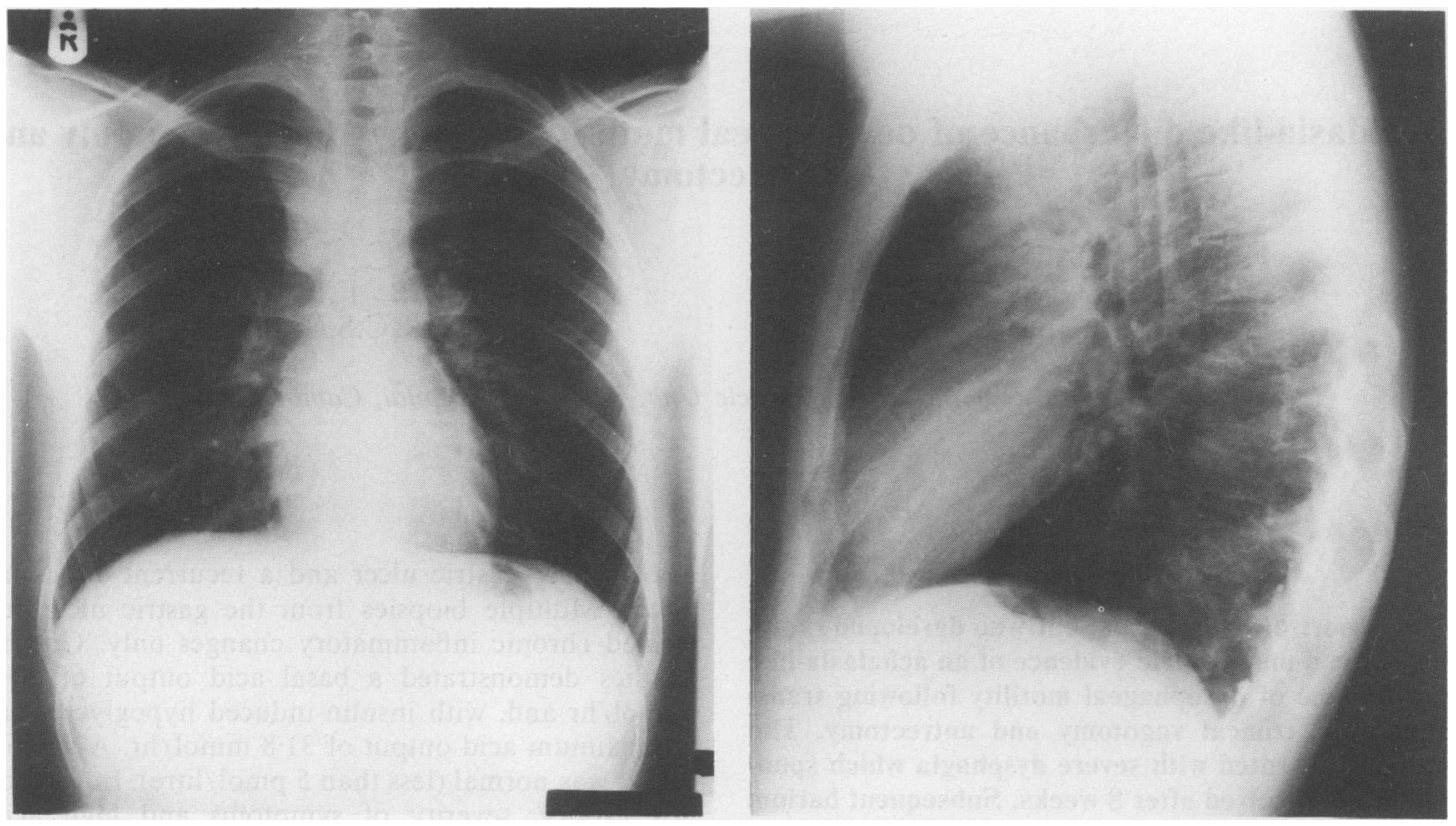

FIG. 1. Posterior-anterior and lateral chest $\mathrm{X}$-rays showing mediastinal mass seen to lie anteriorly.

of afferent fibres in the vagus nerve although deformation of the airways due to the presence of tumour in the mediastinum is also a possible mechanism.

Although they may be solitary, $70 \%$ of neurofibrosarcomas occur in association with neurofibromatosis, and the reported overall incidence of neurofibrosarcoma complicating neurofibromatosis varies from 13-29\% (Brasfield and Das Gupta, 1972; Storm et al., 1980). Neurofibrosarcoma most commonly occurs in the extremities arising from a peripheral nerve. It is not clear whether malignant change always develops in a pre-existing neurofibroma or whether neurofibrosarcoma occurs de novo.

The prognosis and treatment of neurofibrosarcoma depends upon the histological grade of tumour, the degree of local invasion and the presence or absence of metastases at the time of presentation. Knowledge of the natural history and best method of treatment for neurofibrosarcoma at any site is hampered by its rarity, but the tumours are usually highly malignant with a propensity for local recurrence and pulmonary metastases. Storm et al. (1980) reviewing experience with peripheral tumours, reported that local excision alone resulted in a local recurrence rate of $66 \%$. This may be reduced to $20 \%$ with radical surgery combined with pre-operative radiotherapy and chemotherapy in the form of intra-arterial adriamycin. Despite control of initial or secondary local disease, however, $44 \%$ of patients died of metastases and the overall projected 5-year survival was $40 \%$. A similar overall 5-year survival has been found in other series
(Wander and Das Gupta, 1977). The prognosis of intrathoracic sarcomas is worse than that of peripheral tumours because of the difficulty of performing a radical resection of the primary tumour and the inability to give regional chemotherapy.

Neurofibrosarcoma of the vagus nerve is thus a rare and highly malignant tumour, probably best treated by radical resection. Local control of disease may be improved with the addition of pre- or postoperative radiotherapy (Storm et al., 1980). Since most patients die of systemic metastases, adjuvant chemotherapy seems logical but no evidence is available for intrathoracic tumours.

\section{Acknowledgments}

I should like to thank Dr G. J. Gibson and Mr C. J. Hilton for permission to report this case.

\section{References}

BRASFIELD, R.D. \& DAS GUPTA, T.K. (1972) Von Recklinghausen's disease. A clinicopathological study. Annals of Surgery, 175, 86.

Russell, W.O., Cohen, J., Enzinger, F., Hasdu, S.I., Heise, H., MARTIN, R.G., MEISSNER, W., Miller, W.T., SCHMitZ, R.L. \& SuIT, H.D. (1977) A clinical and pathological staging system for soft tissue sarcomas. Cancer, 40, 1562.

StORM, F.K., Ellber, F.R., MirRa. J. \& Morton, D.C. (1980) Neurofibrosarcoma. Cancer, 45, 126.

STRICKLAND, B. \& WolverSON, M.K. (1974) Intrathoracic vagus nerve tumours. Thorax, 29, 215.

WANDER, J.V. \& DAS GUPTA, T.K. (1977) Neurofibromatosis. Current Problems in Surgery, 14, 36.

(Accepted 17 June 1982) 